\title{
Drug-susceptibility of isolates of Brachyspira hyodysenteriae isolated from colonic mucosal specimens of pigs collected from slaughter houses in Japan in 2009
}

\author{
Keita KAJIWARA ${ }^{1,2)}$, Midori KOZAWA ${ }^{2)}$, Takuya KANAZAWA ${ }^{2)}$, Kouji UETSUKA ${ }^{2)}$, Hiromi NAKAJIMA ${ }^{2)}$ and \\ Yoshikazu ADACHI ${ }^{2} *$ \\ 1) United Graduate School of Agricultural Science, Tokyo University of Agriculture and Technology, 3-5-8 Fuchu, Tokyo 183-8509, Japan \\ ${ }^{2)}$ School of Agriculture, Ibaraki University, 3-21-1 Ami, Ibaraki 300-0393, Japan
}

(Received 23 October 2015/Accepted 9 November 2015/Published online in J-STAGE 22 November 2015)

ABSTRACT. Twenty nine isolates identified as Brachyspira hyodysenteriae were most susceptible to carbadox and metronidazole, whereas they were resistant to macrolides. The isolates showed intermediate susceptibility to tiamulin, lincomycin, penicillin G, ampicillin, chloramphenicol, tetracycline, enrofloxacin and valnemulin, with $\mathrm{MIC}_{50}$ values ranging from 0.39 to 3.13 .

KEY WORDS: Brachyspira hyodysenteriae, drug-susceptibility, MIC

doi: 10.1292/jvms.15-0608; J. Vet. Med. Sci. 78(3): 517-519, 2016

Drug-susceptibility tests on Brachyspira hyodysenteriae using an agar dilution technique have been reported by Kitai et al. [4], and the results have been applied for the eradication of B. hyodysenteriae from pig herds affected with swine dysentery. Formerly, carbadox and lincomycin had frequently been used, and recently, valnemulin and tiamulin are using for prevention and eradication of swine dysentery. The MICs of carbadox ranged from $0.003>$ to 0.05 and lincomycin ranged from 25 to 100 [12]. Concurrently, drug-resistant $B$. hyodysenteriae, and B. pilosicoli have also been reported $[6,10]$. In this study, we performed drug-susceptibility tests to investigate the MICs and the presence of drug-resistant isolates of $B$. hyodysenteriae isolated from colonic mucosal specimens collected from slaughter houses in 2009. Several Brachyspira species have been isolated from pigs in slaughter houses in the U.S.A. [2], as has B. hyodysenteriae in Japan. In order to obtain relevant minimum inhibitory concentration (MIC) values, the classification of brachyspiral isolates can be important. If the isolates were not classified as B.hyodysenteriae, several species differing from B.hyodysenteriae may be contaminated and in drugsusceptibility tests, the MIC values may be different from the values in the tests using classified isolates. Therefore, we performed drug-susceptibility tests using classified isolates.

The brachyspiral isolation from colonic specimens was performed as follows: Sheep blood trypticase soy agar (TSA, Difco, Detroit, MI, U.S.A.) containing $400 \mu \mathrm{g} / \mathrm{m} l$ spectinomycin (Pfizer, Tokyo, Japan) was used for the anaerobic isolation as described previously [11] using AnaeroPak Kenki (Mitsubishi Gas Chem., Tokyo, Japan). Isolates were

*Correspondence to: Adachi, Y., School of Agriculture, Ibaraki University, 3-21-1 Ami, Ibaraki 300-0393, Japan.

e-mail: yoshikazu.adachi.colobra@vc.ibaraki.ac.jp

(C)2016 The Japanese Society of Veterinary Science

This is an open-access article distributed under the terms of the Creative Commons Attribution Non-Commercial No Derivatives (by-nc-nd) License $<$ http://creativecommons.org/licenses/by-nc-nd/3.0/>. subcultured three times, and the pure isolates were identified as follows: PCR with NOX1 primers using template DNA extracted from the isolates using Instagene ${ }^{\mathrm{TM}}$ Matrix (BIO-RAD, Hercules, CA, U.S.A.) was performed [1]. In addition, the 16S rDNA gene was sequenced after PCR using F3 and R500 primers [3, 8, 9], and the base alignments were compared with those of representative strains obtained from DDBJ. An indole production test was carried out as described previously [8]. Thereafter, the isolates classified as $B$. hyodysenteriae were used for drug-susceptibility tests against 13 antimicrobial agents: carbadox (Pfizer), tiamulin (Novartis Animal Health, Tokyo, Japan), lincomycin (Pharmacia Upjohn, Yokohama, Japan), penicillin G (Meiji Seika Co., Tokyo, Japan), ampicillin (Wako, Osaka, Japan), chloramphenicol (Wako), tetracycline (Wako), erythromycin (Dainippon Pharmaceutical, Osaka, Japan), tylosin (Pfizer), tylvalosin (Bayer, Osaka, Japan), metronidazole (Wako), enrofloxacin (Bayer) and valnemulin (Novartis Animal Health). Drug-susceptibility tests were carried out by an agar dilution technique as described previously [4]. All drugs except for carbadox were diluted by two-fold dilution from 1,000 to $1 \mu \mathrm{g} / \mathrm{m} l$, and $1 \mathrm{~m} l$ of the diluted drugs was mixed with $9 \mathrm{ml}$ of $4 \%$ sheep blood agar. After fixing, the blood agar was used for drug-susceptibility tests. Carbadox was diluted by two-fold dilution from 1,000 to $0.013 \mu \mathrm{g} /$ $\mathrm{ml}$, and blood agars containing carbadox were prepared as mentioned above.

For isolation and identification of B. hyodysenteriae, colonic mucosal specimens of approximately $5 \mathrm{~cm}^{2}$ from 100 pigs were collected from slaughter houses in Ibaraki Prefecture, Japan, in 2009 and kept at $-80^{\circ} \mathrm{C}$. The isolation was performed in 2010 as follows: small mucosal specimens of $2 \mathrm{~mm}^{2}$ from three different regions from each large specimen were cut out, suspended in trypticase soy broth (BBL, U.S.A.) and shaken vigorously. A $25 \mu l$ aliquot of the suspension was inoculated and grown anaerobically. The isolation was qualitative and suitable for the isolation from colonic mucosa without limiting dilution technique, and the growth 
of B.hyodysenteriae was not disturbed by other motile bacteria, i.e. Proteus sp. and, Salmonella sp.. Twenty-nine isolates were identified, and the characteristics were as follows: positive PCR with NOX1 primers, homology greater than $99.7 \%$ with the $16 \mathrm{~S}$ rDNA sequence of representative $B$. hyodysenteriae, $\beta$-hemolysis on blood agar and positive indole production. The base alignments of the $16 \mathrm{~S}$ rDNA of $B$. hyodysenteriae isolates from F3 to R500 were registered in DDBJ (Accession No.LC055432-055461).

For the drug-susceptibility tests, classified B. hyodysenteriae were used, and the results are shown in Tables 1 and 2 . The twenty-nine $B$. hyodysenteriae isolates were most susceptible to carbadox and metronidazole, whereas macrolides, i.e., erythromycin, tylosin and tylvalosin, had no effect on these isolates. Unfortunately, the use of carbadox had been legally prohibited from medicating and preventing pigs, because carbadox is a carcinogenic substance. The isolates showed intermediate susceptibility to tiamulin, lincomycin, penicillin $\mathrm{G}$, ampicillin, chloramphenicol, tetracycline and valnemulin. The $\mathrm{MIC}_{90}$ values ranged from 1.56 to 12.5 . These results using classified isolates may indicate relevant MIC values, and they were similar to the results reported by Uezato et al. [12], but there were several differences, with comparatively higher MICs for tiamulin, and valnemulin than the previous results [12]. After continuous drug-administration for eradication of swine dysentery, the isolates may have acquired drug-resistance. B.hyodysenteriae ATCC 31212 [7] has acquired the resistance to tylosin [5, 6] and erythromycin [6]. The point mutation at base position 2058 of 23S rDNA was observed [6] and was A-T transition mutation [6], whereas strain ATCC27164 was sensitive to tylosin [5], and the MICs were 1.56-12.5 to the drug [5] and any point mutation at base position 2058 was not observed $[6,10]$. Therefore, in the case of macrolides, point mutations in the $23 \mathrm{~S}$ rDNA of $B$. hyodysenteriae play a role in drug resistance and need to be investigated to determine of the mechanism.
Table1. Drug-susceptibility of 29 isolates of Brachyspira hyodysenteriae

\begin{tabular}{lccc}
\hline \multirow{2}{*}{ Antimicrobial agent } & \multicolumn{3}{c}{ MIC $(\mu g / m l)$} \\
\cline { 2 - 4 } & Range & $\mathrm{MIC}_{90}{ }^{\mathrm{a})}$ & $\mathrm{MIC}_{50}{ }^{\mathrm{b})}$ \\
\cline { 2 - 4 } Carbadox & $0.013>-0.05$ & $0.013>$ & $0.013>$ \\
Tiamulin & $0.1-12.5$ & 6.25 & 1.56 \\
Lincomycin & $0.78-25$ & 12.5 & 3.13 \\
Penicillin G & $0.39-6.25$ & 1.56 & 0.39 \\
Ampicillin & $0.1>-6.25$ & 6.25 & 0.39 \\
Chloramphenicol & $1.56-3.13$ & 3.13 & 1.56 \\
Tetracycline & $0.1>-6.25$ & 6.25 & 0.39 \\
Erythromycin & $50-100<$ & $100<$ & $100<$ \\
Tylosin & $0.1>-100<$ & $100<$ & 100 \\
Tylvalosin & $0.39-100$ & 50 & 25 \\
Metronidazole & $0.1>-0.39$ & 0.39 & 0.2 \\
Enrofloxacin & $0.1>-50$ & 25 & 3.13 \\
Valnemulin & $0.39-6.25$ & 6.25 & 3.13 \\
\hline
\end{tabular}

a) MICs of $90 \% \leq$ of the isolates used in these tests. b) MICs of $50 \% \leq$ of the isolates used in these tests.

\section{REFERENCES}

1. Atyeo, R. F., Stanton, T. B., Jensen, N. S., Suriyaarachichi, D. S. and Hampson, D. J. 1999. Differentiation of Serpulina species by NADH oxidase gene (nox) sequence comparisons and noxbased polymerase chain reaction tests. Vet. Microbiol. 67: 47-60. [Medline] [CrossRef]

2. Burrough E. R. 2013. Swine dysentery - Re-emergence in the United States and Canada. In: Proceedings from 6th International Conference on Coloinic Spirochaetal Infection in Animals and Humans.

3. Fellström, C., Pettersson, B., Thomson, J., Gunnarsson, A., Persson, M. and Johanssen, K. E. 1997. Identification of Serpulina species associated with porcine colitis by biochemical analysis and PCR. J. Clin. Microbiol. 35: 462-467. [Medline]

4. Kitai, K., Kashiwazaki, M., Adachi, Y., Kume, T. and Arakawa, A. 1979. In vitro activity of 39 antimicrobial agents against Treponema hyodysenteriae. Antimicrob. Agents Chemother. 15:

Table 2. Distribution of each MIC of B.hyodysenteriae isolates depending on drug concentration

\begin{tabular}{lccccccccccccc}
\hline \multirow{2}{*}{ Antimicrobial agents } & \multicolumn{10}{c}{ Distribution of each $\mathrm{MIC}(\mu \mathrm{g} / \mathrm{m} l)$} \\
\cline { 2 - 15 } & $0.1>$ & 0.1 & 0.2 & 0.39 & 0.78 & 1.56 & 3.13 & 6.25 & 12.5 & 25 & 50 & 100 & $100<$ \\
\hline Carbadox & $\left.29^{a}\right)$ & 0 & 0 & 0 & 0 & 0 & 0 & 0 & 0 & 0 & 0 & 0 & 0 \\
Tiamulin & 0 & 0 & 0 & 1 & 11 & 11 & 1 & 4 & 1 & 0 & 0 & 0 & 0 \\
Lincomycin & 2 & 0 & 0 & 0 & 7 & 3 & 3 & 3 & 10 & 1 & 0 & 0 & 0 \\
Penicillin G & 0 & 0 & 3 & 12 & 6 & 7 & 0 & 1 & 0 & 0 & 0 & 0 & 0 \\
Ampicillin & 5 & 0 & 3 & 11 & 1 & 2 & 2 & 5 & 0 & 0 & 0 & 0 & 0 \\
Chloramphenicol & 0 & 0 & 0 & 0 & 0 & 25 & 2 & 2 & 0 & 0 & 0 & 0 & 0 \\
Tetracycline & 7 & 0 & 0 & 9 & 7 & 1 & 1 & 4 & 0 & 0 & 0 & 0 & 0 \\
Erythromycin & 0 & 0 & 0 & 0 & 0 & 0 & 0 & 0 & 0 & 2 & 3 & 3 & 21 \\
Tylosin & 0 & 0 & 0 & 0 & 0 & 0 & 0 & 0 & 1 & 4 & 4 & 15 & 5 \\
Tylvalosin & 0 & 0 & 0 & 1 & 0 & 2 & 6 & 2 & 3 & 9 & 5 & 1 & 0 \\
Metronidazole & 6 & 0 & 15 & 8 & 0 & 0 & 0 & 0 & 0 & 0 & 0 & 0 & 0 \\
Enrofloxacin 7 & 7 & 0 & 0 & 1 & 1 & 0 & 6 & 8 & 1 & 3 & 2 & 0 & 0 \\
Valnemulin & 1 & 0 & 1 & 2 & 1 & 4 & 10 & 10 & 0 & 0 & 0 & 0 & 0 \\
\hline
\end{tabular}

a) Range of carbadox was $0.013>-0.05$. 
392-395. [Medline] [CrossRef]

5. Kitai, K., Kashiwazaki, M., Adachi, Y., Kunugita, K. and Arakawa, A. 1987. In vitro antimicrobial activity against reference strains and field isolates of Treponema hyodysenteriae. Antimicrob. Agents Chemother. 31: 1935-1938. [Medline] [CrossRef]

6. Karlsson, M., Fellström, C., Heltander, M. U. K., Johansson, K.E. and Franklin, A. 1999. Genetic basis of macrolide and lincosamide resistance in Brachyspira (Serpulina) hyodysenteriae. FEMS Microbiol. Lett. 172: 255-260. [Medline] [CrossRef]

7. Kinyon, J. M., Hrris, D. L. and Glock, R. D. 1977. Enteropathogenicity of various isolates of Treponema hyodysenteriae. Infect. Immun. 15: 638-646. [Medline]

8. Ochiai, S., Adachi, Y. and Mori, K. 1997. Unification of the general Serpulina and Brachyspira, and proposal of Brachyspira hyodysenteriae comb. nov., Brachyspira innocens comb. nov. and Brachyspira pilosicoli comb. nov. Microbiol. Immunol. 41: 445-452. [Medline] [CrossRef]
9. Park, N. Y., Chung, C. Y., Mclaren, A. J., Atyeo, R. F. and Hampson, D. J. 1995. Polymerase chain reaction for identification of human and porcine spirochetes recovered from cases of intestinal spirochaetosis. FEMS Microbiol. Lett. 125: 225-229. [Medline] [CrossRef]

10. Prapasarakul, N., Ochi, K. and Adachi, Y. 2003. In vitro susceptibility and a new point mutation associated with tylosinresistance in Japanese canine intestinal spirochetes. J. Vet. Med. Sci. 65: 1275-1280. [Medline] [CrossRef]

11. Songer, J. G., Kinyon, J. M. and Harris, D. L. 1976. Selective medium for isolation of Treponema hyodysenteriae. J. Clin. Microbiol. 4: 57-60. [Medline]

12. Uezato, K., Kinjo, E. and Adachi, Y. 2004. In vitro susceptibility of 37 isolates of Brachyspira hyodysenteriae isolated from pigs in Okinawa Prefecture to 21 antimicrobial agents. J. Vet. Med. Sci. 66: 307-309. [Medline] [CrossRef] 